\title{
High-Energy Limit of Quantum Electrodynamics beyond Sudakov Approximation
}

\author{
Alexander A. Penin ${ }^{* \dagger}$ \\ Department of Physics, University of Alberta, Edmonton AB T6G 2J1, Canada \\ E-mail: penindualberta.ca
}

\begin{abstract}
We study the high-energy behavior of the scattering amplitudes in quantum electrodynamics beyond the leading order of the small electron mass expansion in the leading logarithmic approximation. In contrast to the Sudakov logarithms, the mass-suppressed double-logarithmic radiative corrections are induced by a soft electron pair exchange and result in enhancement of the powersuppressed contribution. Possible applications of our result to the analysis of the high-energy processes is also discussed.
\end{abstract}

12th International Symposium on Radiative Corrections (Radcor 2015) and LoopFest XIV (Radiative Corrections for the LHC and Future Colliders)

15-19 June 2015

UCLA Department of Physics \& Astronomy Los Angeles, CA, USA

\footnotetext{
*Speaker.

${ }^{\dagger}$ The talk is based on Ref. [四]
} 
In a renowned paper [2] V.V. Sudakov derived the leading asymptotic behavior of an electron scattering amplitude in quantum electrodynamics (QED) at high energy. It is determined by the "Sudakov" radiative corrections, which include the second power of the large logarithm of the electron mass $m_{e}$ divided by a characteristic momentum transfer of the process per each power of the fine structure constant $\alpha$. Sudakov double logarithms exponentiate and result in a strong universal suppression of any electron scattering amplitude with a fixed number of emitted photons in the limit when all the kinematic invariants of the process are large. This result plays a fundamental role in particle physics. Within different approaches it has been extended to the nonabelian

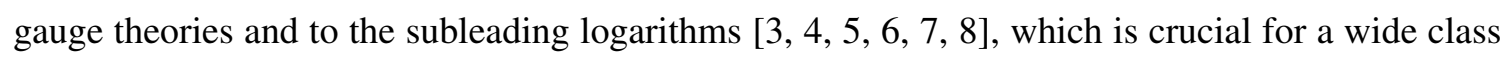
of applications from deep inelastic scattering to Drell-Yan processes and the Higgs boson production. At the same time no significant progress has been achieved in the study of the logarithmically enhanced corrections to the subleading contributions suppressed by a power of electron mass at high energies. However, the power-suppressed contributions are of great interest. They can become asymptotically dominant at very high energies due to Sudakov suppression of the leading terms. At the intermediate energies the power corrections in many cases are phenomenologically

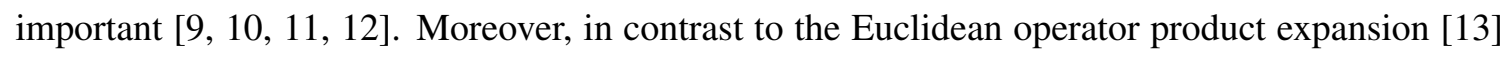
or nonrelativistic threshold dynamics [14] very little is known about the general all-order structure of the large logarithms beyond the leading-power approximation in the high-energy limit, which is a real challenge for the effective field theory approach. This problem is now actively discussed in various contexts (see e.g. [15, [6, [17, 8, ㅁ, [20]). In this Letter we make the first step toward the solution of the problem and generalize the result of Ref. [] to the leading power-suppressed contribution. We present a detailed analysis of the electron scattering in the external field and later discuss the extension of the result to more complex processes.

The amplitude $\mathscr{F}$ of the electron scattering in an external field can be parameterized in the standard way by the Dirac and Pauli form factors

$$
\mathscr{F}=\bar{\psi}\left(p_{1}\right)\left(\gamma_{\mu} F_{1}+\frac{i \sigma_{\mu v} q^{v}}{2 m_{e}} F_{2}\right) \psi\left(p_{2}\right)
$$

The Pauli form factor $F_{2}$ does not contribute in the approximation discussed in this Letter and we mainly focus on the high-energy behavior of the Dirac form factor $F_{1}$. We consider the limit of the on-shell electron $p_{1}^{2}=p_{2}^{2}=m_{e}^{2}$ and the large Euclidean momentum transfer $Q^{2}=-\left(p_{2}-p_{1}\right)^{2}$ when the ratio $\rho \equiv m_{e}^{2} / Q^{2}$ is positive and small. The Dirac form factor can then be expanded in an asymptotic series in $\rho$

$$
F_{1}=S_{\lambda} \sum_{n=0}^{\infty} \rho^{n} F_{1}^{(n)}
$$

where $F_{1}^{(n)}$ are given by the power series in $\alpha$ with the coefficients depending on $\rho$ only logarithmically. The factor $S_{\lambda}=\exp \left[-\frac{\alpha}{2 \pi} B(\rho) \ln \left(\lambda^{2} / m_{e}^{2}\right)\right]$ with $B(\rho)=\ln \rho+\mathscr{O}(1)$ accounts for the universal singular dependence of the amplitude on the auxiliary photon mass $\lambda$ introduced to regulate the infrared divergences [ [R]]. In the double-logarithmic approximation the leading term is given by the Sudakov exponent $F_{1}^{(0)}=e^{-x}$, with $x=\frac{\alpha}{4 \pi} \ln ^{2} \rho$ [22]. Let us outline our approach for the analysis of the power-suppressed double-logarithmic contributions. We use the expansion by regions method [23, [24, [25] to get a systematic expansion of the Feynman integrals in $\rho$. In this method the coefficients $F_{1}^{(n)}$ are given by the sum over contributions of different virtual momentum regions. 


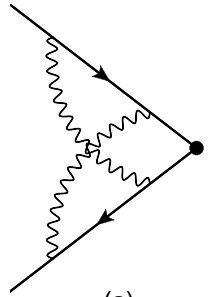

(a)

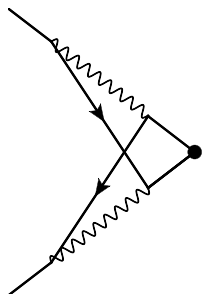

(b)

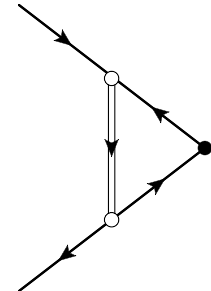

(c)

Figure 1: Different representations of the two-loop Feynman diagram giving the leading power-suppressed double-logarithmic contribution. In figure (c) the double line arrow represents the soft electron pair propagator and the empty blobs represent the nonlocal interaction of the soft electron pair to the eikonal electrons and positrons.

Each contribution is represented by a Feynman integral which in general is divergent. These spurious divergences result from the process of scale separation and have to be dimensionally regulated. The singular terms cancel out in the sum of all regions but can be used to determine the logarithmic contributions to $F_{1}^{(n)}$. The double-logarithmic contributions are determined by the leading singular behavior of the integrals and can be found by the method developed in Refs. [2], 22], 26]. Though the method is blind to the power corrections, it can be applied in this case since the expansion by regions provides the integrals which are homogeneous in the expansion parameter. Sudakov logarithms are produced by the soft virtual photons, which are collinear to either $p_{1}$ or $p_{2}$. We have found that such a configuration of virtual momenta does not produce double logarithms in the first order in $\rho$. This observation agrees with the analysis [27] of the cusp anomalous dimension, which determines the double-logarithmic corrections to the light-like Wilson line with a cusp. For the large cusp angle corresponding to the limit $\rho \rightarrow 0$ from the result of Ref. [27] one gets

$$
\Gamma_{\text {cusp }}=-\frac{\alpha}{\pi} \ln \rho\left(1+\mathscr{O}\left(\rho^{2}\right)\right),
$$

with vanishing first-order term in $\rho$. Nevertheless, the $\mathscr{O}(\rho)$ double-logarithmic contribution does exist but originates from completely different virtual momentum configuration described below. Let us consider an electron propagator $S=\frac{\not p_{i}-l+m_{e}}{\left(p_{i}-l\right)^{2}-m_{e}^{2}}$, where $l$ is the momentum of a virtual photon with the propagator $D=\frac{-g_{\mu \nu}}{l^{2}-\lambda^{2}}$. In the soft-photon limit $l \rightarrow 0$ the electron propagator becomes eikonal $S \approx-\frac{p_{i}+m_{e}}{2 p_{i} l}$ and develops a collinear singularity when $l$ is parallel to $p_{i}$. Alternatively, we may consider the soft-electron limit $l^{\prime} \rightarrow 0$, where $l^{\prime}=p_{i}-l$. Then the electron propagator becomes scalar $S \approx \frac{m_{e}}{l^{2}-m_{e}^{2}}$ while the photon propagator becomes eikonal $D \approx \frac{g_{\mu v}}{2 p_{i} l^{\prime}-m_{e}^{2}+\lambda^{2}}$. Thus the roles of the electron and photon propagators are exchanged. The existence of non-Sudakov doublelogarithmic contributions due to soft electron exchange has actually been known for a long time [26, [28]. However in our case this virtual momentum configuration does not produce a doublelogarithmic contribution in one loop because the momentum shift distorts the eikonal structure of the second electron propagator and removes the soft singularity at small $l^{\prime}$ necessary to get the second power of the large logarithm.

This may be avoided only in the two-loop diagram of nonplanar topology, Fig. W(a). After shifting the photon virtual momenta by $p_{1}$ and $p_{2}$ the diagram can be twisted into the shape of 


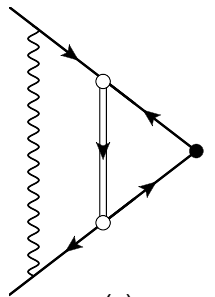

(a)

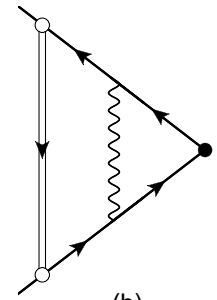

(b)

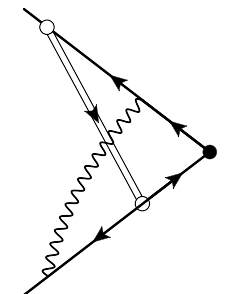

(c)

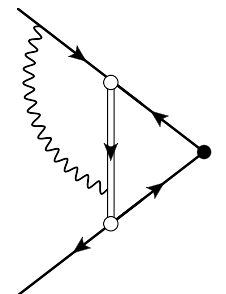

(d)

Figure 2: Feynman diagrams contributing to the double-logarithmic correction factors $\phi^{a, b, c, d}$, Eq. (पD).

Fig. $\llbracket(b, c)$ with soft electron pair exchange between the eikonal lines. The corresponding contribution has an explicit suppression factor $m_{e}^{2}$ from two soft electron propagators. Hence the integration over the virtual momenta can be performed in the leading order of the small electron mass expansion. Note that in the case under consideration the electron mass regulates both soft and collinear divergences and we can put $\lambda=0$. The calculation is conveniently performed by using the light-cone coordinates where $p_{1} \approx p_{1-}$ and $p_{2} \approx p_{2+}$. In this representation only the interaction of the transverse photons to soft electrons is not mass-suppressed and we can use $\frac{g_{k l}}{2 p_{i} l}$ for the eikonal photon propagators. To get the double-logarithmic part of the correction we use Sudakov parametrization of a virtual photon momentum $l=u p_{1}+v p_{2}+l_{\perp}$. After integrating over the transverse components $l_{\perp}$ we get the following representation of the two-loop power-suppressed form factor

$$
\left.F_{1}^{(1)}\right|_{2-\text { loop }}=-4 x^{2} \int K\left(\eta_{1}, \eta_{2}, \xi_{1}, \xi_{2}\right) \mathrm{d} \eta_{1} \mathrm{~d} \eta_{2} \mathrm{~d} \xi_{1} \mathrm{~d} \xi_{2}
$$

where $\eta=\ln v / \ln \rho, \xi=\ln u / \ln \rho$, the integration goes over the four-dimensional cube $0<\eta_{i}, \xi_{i}<$ 1 , and the kernel

$$
\begin{aligned}
K\left(\eta_{1}, \eta_{2}, \xi_{1}, \xi_{2}\right)= & \theta\left(1-\eta_{1}-\xi_{1}\right) \theta\left(1-\eta_{2}-\xi_{2}\right) \\
& \times \theta\left(\eta_{2}-\eta_{1}\right) \theta\left(\xi_{1}-\xi_{2}\right)
\end{aligned}
$$

selects the kinematically allowed region of double-logarithmic integration. This gives $F_{1}^{(1)}=$ $-\frac{x^{2}}{3}+\mathscr{O}\left(x^{3}\right)$, in agreement with [0, 29]. The higher-order double-logarithmic corrections are generated in a usual way through the exchange of soft photons with the propagator $\frac{-g_{+-}}{l^{2}-\lambda^{2}}$. The relevant topologies of the three-loop diagrams are given in Fig. $\square$. Note that the soft photon exchange between the soft and internal eikonal electron lines does not produce the double-logarithmic correction. By using the factorization properties of the soft photon contribution [] we find the following representation of the all-order double-logarithmic result

$$
\begin{aligned}
F_{1}^{(1)}= & -4 x^{2} \int \phi^{b}\left(\eta_{1}, \xi_{2}\right) \phi^{c}\left(\eta_{1}, \xi_{1}\right) \phi^{c}\left(\xi_{2}, \eta_{2}\right) \phi^{d}\left(\eta_{2}\right) \phi^{d}\left(\xi_{1}\right) \\
& \times\left[\phi^{a}\left(\eta_{2}, \xi_{1}\right) K_{1}\left(\eta_{1}, \eta_{2}, \xi_{1}, \xi_{2}\right)\right. \\
& \left.+K_{2}\left(\eta_{1}, \eta_{2}, \xi_{1}, \xi_{2}\right)\right] \mathrm{d} \eta_{1} \mathrm{~d} \eta_{2} \mathrm{~d} \xi_{1} \mathrm{~d} \xi_{2},
\end{aligned}
$$

where the Sudakov correction factors corresponding to Fig. [D(a-d) are

$$
\phi^{a}(\eta, \xi)=\exp \left[-x(1-\eta-\xi)^{2}\right]
$$




\begin{tabular}{c|c|c|c|c|c|c|c}
$n$ & 1 & 2 & 3 & 4 & 5 & 6 & 7 \\
\hline$(-1)^{n} n ! c_{n}$ & $\frac{29}{30}$ & $\frac{257}{210}$ & $\frac{1231}{630}$ & $\frac{396581}{103950}$ & $\frac{5531381}{630630}$ & $\frac{72078311}{3153150}$ & $\frac{4510839803}{68918850}$
\end{tabular}

Table 1: The normalized coefficients of the series $(\mathbb{Q})$ up to $n=7$.

$$
\begin{aligned}
\phi^{b}(\eta, \xi) & =\exp [-2 x \eta \xi], \\
\phi^{c}(\eta, \xi) & =\exp [-x \eta(\eta+2 \xi-2)], \\
\phi^{d}(\eta) & =\exp \left[-x(1-\eta)^{2}\right],
\end{aligned}
$$

respectively, and the new kernels read

$$
\begin{aligned}
K_{1}\left(\eta_{1}, \eta_{2}, \xi_{1}, \xi_{2}\right)= & \theta\left(1-\eta_{2}-\xi_{1}\right) \theta\left(1-\eta_{2}-\xi_{2}\right) \\
& \times \theta\left(\eta_{2}-\eta_{1}\right) \theta\left(\xi_{1}-\xi_{2}\right), \\
K_{2}\left(\eta_{1}, \eta_{2}, \xi_{1}, \xi_{2}\right)= & \theta\left(1-\eta_{1}-\xi_{1}\right) \theta\left(1-\eta_{2}-\xi_{2}\right) \\
& \times \theta\left(\eta_{2}-\eta_{1}\right) \theta\left(\xi_{1}+\eta_{2}-1\right),
\end{aligned}
$$

with $K=K_{1}+K_{2}$. We are not able to find the result for the four-fold integral (G) in a closed analytic form. However, the coefficients of the series

$$
F_{1}^{(1)}=-\frac{x^{2}}{3}\left(1+\sum_{n=1}^{\infty} c_{n} x^{n}\right)
$$

can in principle be analytically computed for any given $n$ corresponding to the $(n+2)$-loop doublelogarithmic contribution. The first seven coefficients of the series are listed in Table $\mathbb{U}$. The series (Q) is useful only for sufficiently small $x$. For large $x$ the integral (G) can be computed numerically. The result of the numerical evaluation is presented in Fig. [3 for the function $f(x)=$ $-3 F_{1}^{(1)}$. The function rapidly grows at $x \sim 1$ and then monotonically approaches the limit $f(\infty)=$ $1.33496 \ldots$ corresponding to

$$
F_{1}^{(1)}(x=\infty)=-0.444988 \ldots
$$

Thus the power-suppressed amplitude is enhanced by the double-logarithmic corrections at high energy. A similar effect has been observed before e.g. for the electron-muon backward scattering [R6]. In the case under consideration the enhancement may be related to a specific structure of the process with the soft electron pair exchange. Through the pair emission an eikonal electron is converted into an eikonal positron with approximately the same momentum but opposite electric charge, Fig. W(c). As a result the double-logarithmic contribution of the topology of Fig. $\nabla(c)$ has an opposite sign with respect to the one of Fig. W(a,b,d) and cancels the standard Sudakov suppression. The double-logarithmic approximation of $F_{1}^{(1)}$ given above is valid for $\alpha \ll x \ll 1 / \alpha$, which covers the energy interval $1 \ll|\ln \rho| \ll 1 / \alpha$ sufficient for any practical applications. For higher energies corresponding to $x \sim 1 / \alpha$ the subleading terms proportional to powers of $\alpha \ln \rho \sim 1$ have to be resumed to all orders.

Unlike the Sudakov double logarithms, the leading power-suppressed double-logarithmic corrections depend not only on the charges of the initial and final states but also on the details of the scattering process. For example, the non-singlet (i.e. without a closed electron loop) $\mathscr{O}(\rho)$ 


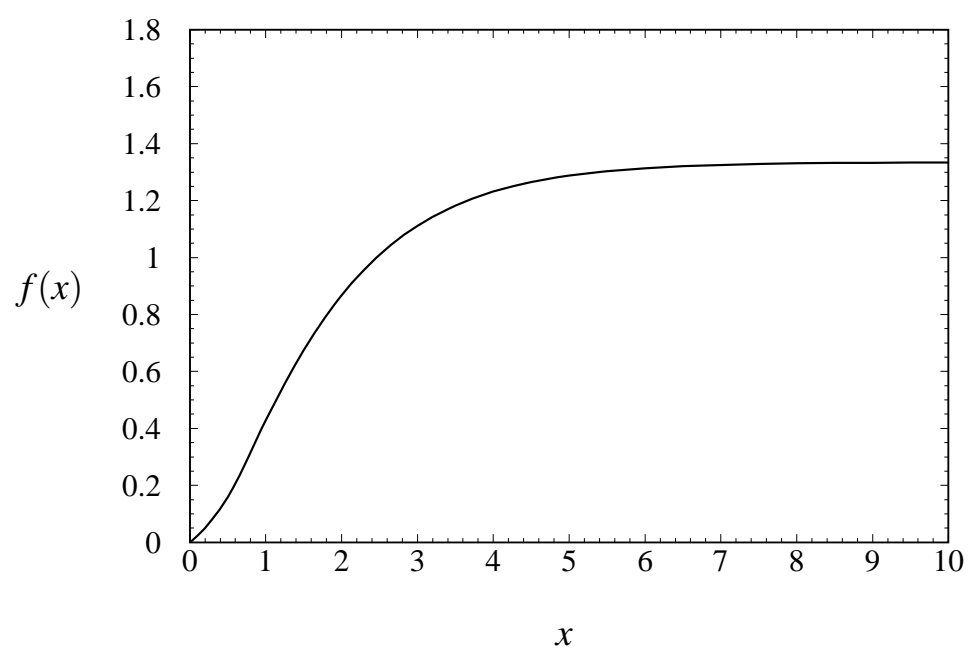

Figure 3: The result of the numerical evaluation of the function $f(x)=-3 F_{1}^{(1)}$.

double-logarithmic corrections to the scalar form factor vanish to all orders in $\alpha$ due to a specific Lorentz and Dirac structure of the soft electron pair interaction with the eikonal electrons and positrons. A less trivial example is the Pauli form factor. The expansion of $F_{2}$ in $\rho$ (cf. Eq. (D)) starts with the first order term $F_{2}^{(1)}$. In the double-logarithmic approximation $F_{2}^{(1)}=0$ and for the leading mass correction from the soft electron pair exchange we obtain $F_{2}^{(2)}=4 F_{1}^{(1)}$, in agreement with [Q, 29]. Thus the $\mathscr{O}(\rho)$ corrections are universally related to the soft electron pair exchange and can be be obtained as a straightforward generalization of our analysis for more complicated processes such as Bhabha scattering, where only the leading result of the small electron mass expansion is available in two loops [B], [B]]. Moreover, up to two loops the structure of the $\mathscr{O}(\rho)$ double-logarithmic correction in quantum chromodynamics (QCD) is similar to the one in QED. In particular, the double-logarithmic power-suppressed term in two-loop corrections to the heavyquark vector form factor differs from the QED result only by the $C_{F}^{2}-C_{A} C_{F} / 2$ color factor of the diagram in Fig. W. Thus our method can be applied to the calculation of the dominant two-loop power-suppressed corrections to the high-energy processes involving heavy quarks. For the energies ranging from approximately 10 to 100 times the heavy-quark mass we have $|\ln \rho| \gg 1$ and $\rho \ln ^{4} \rho \sim 1$, i.e. the double-logarithmic terms saturate the power-suppressed contribution and are comparable in magnitude to the nonlogarithmic leading-power corrections in the strong coupling constant, which are phenomenologically significant. Beyond the two-loop approximation our result is not directly applicable to the QCD amplitudes since the eikonal gluons in Fig. W(b) can radiate soft gluons producing additional double-logarithmic corrections. As a consequence, the leading power-suppressed double-logarithmic corrections to the heavy-quark vector form factor get a nonabelian contribution in every order of perturbation theory in contrast to the purely abelian Sudakov double logarithms. The method could also be applied to the analysis of the two-loop electroweak corrections at high energy in the spirit of [32, [3], [34, [35, [36]

To summarize, we have generalized the result of Sudakov [ [ $]$ ] to the leading power-suppressed contribution. This is an important step towards a systematic renormalization group analysis of the 
high energy behavior of the gauge theory amplitudes beyond the leading power approximation. The leading power-suppressed double-logarithmic corrections reveal a few characteristic features which distinguish them from the Sudakov double logarithms. In particular, they are induced by a soft electron pair exchange and result in an enhancement of the power-suppressed contribution. In QCD our method can be used for the analysis of the high-energy processes involving heavy quarks up to two loops. Extending the analysis to the higher orders of perturbative QCD and to subleading logarithms is a very interesting problem which is beyond the scope of this Letter.

\section{References}

[1] A. A. Penin, Phys. Lett. B 745, 69 (2015).

[2] V. V. Sudakov, Sov. Phys. JETP 3, 65 (1956) [Zh. Eksp. Teor. Fiz. 30, 87 (1956)].

[3] J. Frenkel and J. C. Taylor, Nucl. Phys. B 116, 185 (1976).

[4] A. H. Mueller, Phys. Rev. D 20, 2037 (1979).

[5] J. C. Collins, Phys. Rev. D 22, 1478 (1980).

[6] A. Sen, Phys. Rev. D 24, 3281 (1981).

[7] G. F. Sterman, Nucl. Phys. B 281, 310 (1987).

[8] S. Catani, Phys. Lett. B 427, 161 (1998).

[9] W. Bernreuther, R. Bonciani, T. Gehrmann, R. Heinesch, T. Leineweber, P. Mastrolia and E. Remiddi, Nucl. Phys. B 706, 245 (2005).

[10] W. Bernreuther, R. Bonciani, T. Gehrmann, R. Heinesch, P. Mastrolia and E. Remiddi, Phys. Rev. D 72, 096002 (2005).

[11] R. Bonciani, A. Ferroglia and A. A. Penin, Phys. Rev. Lett. 100, 131601 (2008).

[12] R. Bonciani, A. Ferroglia and A. A. Penin, JHEP 0802, 080 (2008).

[13] K. G. Wilson, Phys. Rev. 179, 1499 (1969).

[14] W. E. Caswell and G. P. Lepage, Phys. Lett. B 167, 437 (1986).

[15] B. Feucht, J. H. Kuhn, A. A. Penin and V. A. Smirnov, Phys. Rev. Lett. 93, 101802 (2004).

[16] E. Laenen, L. Magnea, G. Stavenga and C. D. White, JHEP 1101, 141 (2011).

[17] T. Becher and G. Bell, Phys. Rev. Lett. 112, 182002 (2014).

[18] A. Banfi, P. F. Monni and G. Zanderighi, JHEP 1401, 097 (2014).

[19] D. de Florian, J. Mazzitelli, S. Moch and A. Vogt, JHEP 1410, 176 (2014).

[20] C. Anastasiou, C. Duhr, F. Dulat, E. Furlan, T. Gehrmann, F. Herzog and B. Mistlberger, JHEP 1503, 091 (2015).

[21] D. R. Yennie, S. C. Frautschi and H. Suura, Annals Phys. 13, 379 (1961).

[22] R. Jackiw, Annals Phys. 48, 292 (1968).

[23] M. Beneke and V. A. Smirnov, Nucl. Phys. B 522, 321 (1998).

[24] V. A. Smirnov, Phys. Lett. B 404, 101 (1997). 
[25] V. A. Smirnov, Applied asymptotic expansions in momenta and masses, Springer Tracts Mod. Phys. 177 (2002) 1.

[26] V. G. Gorshkov, V. N. Gribov, L. N. Lipatov and G. V. Frolov, Sov. J. Nucl. Phys. 6, 95 (1968) [Yad. Fiz. 6, 129 (1967)].

[27] G. P. Korchemsky and A. V. Radyushkin, Nucl. Phys. B 283, 342 (1987).

[28] V. S. Fadin, V. A. Khoze and A. D. Martin, Phys. Rev. D 56, 484 (1997).

[29] P. Mastrolia and E. Remiddi, Nucl. Phys. B 664, 341 (2003).

[30] A. A. Penin, Phys. Rev. Lett. 95, 010408 (2005).

[31] A. A. Penin, Nucl. Phys. B 734, 185 (2006).

[32] B. Jantzen, J. H. Kuhn, A. A. Penin and V. A. Smirnov, Phys. Rev. D 72, 051301 (2005); Phys. Rev. D 74, 019901 (2006).

[33] B. Jantzen, J. H. Kühn, A. A. Penin and V. A. Smirnov, Nucl. Phys. B 731, 188 (2005); Nucl. Phys. B 752, 327 (2006).

[34] J. H. Kühn, F. Metzler and A. A. Penin, Nucl. Phys. B 795, 277 (2008); Nucl. Phys. B 818, 135 (2009).

[35] J. H. Kühn, F. Metzler, A. A. Penin, and S. Uccirati, JHEP 1106, 143 (2011).

[36] A. A. Penin and G. Ryan, JHEP 1111, 081 (2011). 